\title{
On the relationship between fractal geometry of space and time in which a system of interacting cells exists and dynamics of gene expression ${ }^{\star}$
}

\author{
Przemysław Waliszewski ${ }^{1,2 \bowtie}$, Marcin Molski ${ }^{1}$ and Jerzy Konarski ${ }^{1}$ \\ ${ }^{1}$ Department of Theoretical Chemistry, Adam Mickiewicz University, Poznań, Poland; \\ ${ }^{2}$ Department of Medicine, Mount Sinai School of Medicine, New York, U.S.A.
}

Received: 15 October, 2000; revised: 5 February, 2001; accepted: 28 February, 2001

Key words: complexity, fractal, fractal dimension, differentiation, neoplasia

\begin{abstract}
We report that both space and time, in which a system of interacting cells exists, possess fractal structure. Each single cell of the system can restore the hierarchical organization and dynamic features of the entire tumor. There is a relationship between dynamics of gene expression and connectivity (i.e., interconnectedness which denotes the existence of complex, dynamic relationships in a population of cells leading to the emergence of global features in the system that would never appear in a single cell existing out of the system). Fractal structure emerges owing to non-bijectivity of dynamic cellular network of genes and their regulatory elements. It disappears during tumor progression. This latter state is characterized by damped dynamics of gene expression, loss of connectivity, loss of collectivity (i.e., capability of the interconnected cells to interact in a common mode), and metastatic phenotype. Fractal structure of both space and time is necessary for a cellular system to self-organize. Our findings indicate that results of molecular studies on gene expression should be interpreted in terms of space-time geometry of the cellular system. In particular, the dynamics of gene expression in cancer cells existing in a malignant tumor is not identical with the dynamics of gene expression in the same cells cultured in the monolayer system.
\end{abstract}

The nature of space or time in which a complex system of interacting cells exists have been neglected in cellular or molecular research. It has been assumed tacitly that space possesses fea- tures of the Euclidean space (i.e., three dimensions), and time is linear. In consequence, features of space-time in which a biological system performs its functions have not been considered

\footnotetext{
ॠ This paper was presented in part to the International Symposium "Fractals 2000 in Biology and Medicine" in Ascona, Switzerland, 2000.

${ }^{凶}$ Address for correspondence: Przemysław Waliszewski, Os. Jana III Sobieskiego 41 m. 21, 60-688 Poznań, Poland; phone: (48 61) 825 1750; e-mail: WaliszP@amu.edu.pl.

Abbreviations: atRA, all-trans retinoic acid; $\mathrm{P} 19^{\text {mono }}$, murine teratocarcinoma-derived retinoid-sensitive cells grown in the monolayer culture; RAC65 ${ }^{\text {mono }}$, murine teratocarcinoma-derived retinoid-resistant cells grown in the monolayer culture; P19 $9^{\text {agg }}$ and RAC65 ${ }^{\text {agg }}$ stand for cells aggregated in the form of colonies in suspension, treated with atRA for $96 \mathrm{~h}$, and, then, plated out and cultured in the monolayer system.
} 
as important factors influencing gene expression, intercellular signaling, dynamics of growth, or organization of cells into structures of higher order, such as rosettes, cords, bundles, plaques, or glands. Yet, a relationship between genotype and phenotype is non-bijective (i.e., a gene can contribute to the emergence of more than just one phenotypic trait or a phenotypic trait can be determined by expression of several genes) [1]. Since the only bijective relationship is a linear projection, this implies non-linearity of the dynamic cellular network of genes and their protein regulatory elements, (i.e., lack of a proportional relationship between input and outcome). Nonbijectivity of a dynamic cellular network also implies its complexity, (i.e. emergence of the hierarchical network of multiple cross-interacting elements which is sensitive to initial conditions, possesses multiple equilibria, organizes spontaneously into different morphological patterns, and is controled in a dispersed rather than centralized manner), quasi-determinism (i.e., co-existence of deterministic and non-deterministic events), and fractal structure of both space and time in which such a system exists [1].

Three-dimensional Euclidean geometry approximates the geometry of nature [2-4]. Natural objects frequently possess fractal dimension or both fractal dimension and self-similarity. The dimension of such objects on a plane is not equal to 1 or 2 , as we used to think of. It can be a real number lower than 1 (e.g., 0.2619 for the Koch curve, 0.6309 for the Cantor set), between 1 and 2 (e.g., 1.8929 for the Sierpinski carpet) [5], or, under certain circumstances, even greater than 2 (e.g., if spacetime is curved, i.e., space in which rules of classical geometry does not apply). Fractal dimension for biological hierarchical systems, such as cells or tissues is usually between 1.5 and 2.5; however, any real number can occur [1, 5-10]. In the figurative sense, the existence of fractal dimension denotes that the area of the object with fractal dimension is not directly proportional to the square of the length of its side, $z^{2}$. It is proportional to $\mathrm{z}^{\mathrm{k}}$, where $\mathrm{k}$ is fractal dimension.

According to the most general definition, fractal dimension is a real number equal to the limit of the quotient $\log \mathrm{n}(\varepsilon) / \log (1 / \varepsilon)$ at $\varepsilon \rightarrow 0$, where $\mathrm{n}(\varepsilon)$ is a minimum number of $\mathrm{N}$-dimensional cubes of side $\varepsilon$ necessary to cover the entire object, curve, or set of points. In particular, fractal dimension can be an integer number if this is the limit of the quotient (e.g., Peano curve). Fractal dimension can also be defined by self-similarity of structure as described by the power law scaling of the algebraic form $y=a x^{b}$, in which $y$ is the number of objects in the area covered by a single cube or a circle of the linear grid, $\mathrm{x}$ stands for the linear size of the cube or radius of the circle, $a$ is scaling coefficient, and $b$ is dimension of space occupied by the patterns analyzed. One can expect the co-existence of fractal dimension and self-similarity if the coefficient of nonlinear or linear regression $R$ is greater than 0.95. The latter definition underlies the box counting method; the approach applied in the current study [2].

This study has been undertaken to answer the following questions: 1 . how both fractal dimension and self-similarity change during tumor progression? 2. whether the time in which the system of interacting cells performs its functions, such as gene expression, is non-linear, and could also possess a fractal dimension?

Our findings indicate that cells interacting within a complex system do not proliferate, or differentiate in an utterly random manner, nor form a composition of two or three-dimensional objects. The population of such cells possesses a fractal dimension together with self-similarity. The value of fractal dimension changes along with differentiation of cells or spatial evolution, such as aggregation (i.e., transition from a quasi-twodimensional monolayer system to a quasi-threedimensional system, such as aggregated multicellular colony). This is associated with modification of dynamics of gene expression at the microscopic-scale level of cellular organization. Moreover, expression of genes in time can be a non-linear process. We discovered that the time in which expression occurs also possesses a fractal dimension. Those statistical features (i.e., fractal dimension and self-similarity of both space and time in which the system of interacting cells exists) are lost during tumor progression. Although secondary cancer cells transduce the same retinoid signal and form cellular aggregates, all 
populations of those cells possess an integer (i.e., non-fractal) spatial dimension and damped dynamics of gene expression. It appears that fractal geometry of both space and time is necessary for self-organization to emerge within a system of cells.

\section{MATERIALS AND METHODS}

Cell lines. Two genetically-related cancer cell lines, P19 and RAC65, were used as an in vitro model. Multipotent murine embryonal carcinoma cells P19, when aggregated and treated with all-trans retinoic acid (atRA) or dimethyl sulfoxide $\left(\mathrm{Me}_{2} \mathrm{SO}\right)$, differentiate irreversibly into cells of neuroectodermal origin [11]. The retinoid-induced $\left(10^{-7} \mathrm{M}\right.$ atRA) differentiation of $\mathrm{P} 19^{\text {agg }}$ cells results in the appearance of neurons, astrocytes, and glial cells expressing a number of markers specific for those cell types [12-17]. $\mathrm{P} 19^{\mathrm{mono}}$ cells do not differentiate under those circumstances. RAC65 cells, which originate from P19 cells and represent a more advanced stage of tumor progression [18], possess a dominant negative mutation in the alpha retinoic receptor gene, and a different pattern of expression of some Hox genes than P19 cells [19]. Neither RAC65 ${ }^{\text {mono }}$ nor RAC65 ${ }^{\text {agg }}$ cells can differentiate. Those cells continue to proliferate only. P19 cells and RAC65 cells were cultured in Dulbecco's modified Eagle's medium (DMEM) supplemented with heat-inactivated $10 \%$ fetal calf serum (Gibco BRL, Gaithersburg, MD, U.S.A.). The serum contained negligible amounts of both vitamin $\mathrm{A}$ and all-transretinoic acid. $\mathrm{P} 19$ cells were cultured in $3 \% \mathrm{CO}_{2}$ and RAC65 cells required $5 \% \mathrm{CO}_{2}$.

Cell culture. Both cell lines were seeded directly upon the bottom of 6-well plates at low initial densities (i.e., 1000 cells $/ \mathrm{ml}$ ) [20], cultured until $60 \%$ confluency, treated with $100 \mathrm{nM}$ all-trans retinoic acid for 24 or $48 \mathrm{~h}$ in darkness, subjected to morphometric analysis in the 48th hour of treatment and, then, to isolation of total RNA at those timepoints, as described elsewhere [21]. Alternatively, cells were first maintained in suspension for $96 \mathrm{~h}$ in 60-mm bacterial grade Petri dishes at low initial densities (i.e., 2000 cells $/ \mathrm{ml}$ ) to allow colony formation and co-operation while treated with $1 \mathrm{nM}, 10 \mathrm{nM}$, or $100 \mathrm{nM}$ all-trans retinoic acid. Then, the colonies were treated with $1 \times$ Trypsin-EDTA (Gibco BRL, U.S.A.) for $5 \mathrm{~min}$ at $37^{\circ} \mathrm{C}$ to facilitate detachment of cells, seeded upon 6-well plates, cultured without the retinoid for 48 or $72 \mathrm{~h}$, analyzed morphometrically in the 48th hour (only cells treated with $100 \mathrm{nM}$ atRA), and used for isolation of total RNA at both time points.

Relative RT-PCR. This semi-quantitative assay was performed exactly as described elsewhere $[22,23]$. The method allows the amplification of test and marker cDNAs each to be within the linear portion of the sigmoid PCR curve and optimization of the reaction conditions without the need for a marker with the same concentration and similar physico-chemical sequence parameters as the test cDNA. A number of PCR cycles and PCR conditions were determined in such a way that the amounts of the PCR products were within the linear portion of the PCR curve, (i.e., denaturation: $94^{\circ} \mathrm{C}, 5 \mathrm{~min}$; reverse transcription: $0.3 \mu \mathrm{l}$ AMV reverse transcriptase (Boehringer, Germany) per sample, $50^{\circ} \mathrm{C}, 15 \mathrm{~min}$; PCR cycle: 1 unit of Taq DNA polymerase (Perkin-Elmer, U.S.A.) per sample: denaturation $94^{\circ} \mathrm{C}, 30 \mathrm{~s} ; 55^{\circ} \mathrm{C}, 30 \mathrm{~s}$; elongation $72^{\circ} \mathrm{C}, 1 \mathrm{~min}$ ) [22].

The RNA samples were prepared in triplicates. They were normalized against a retinoid-unresponsive molecular marker (e.g., acidic ribosomal phosphoprotein PO, 36B4) [23]. Twenty microliters of the RT-PCR products were loaded onto $1 \%$ agarose gel in $1 \times$ TAE buffer, separated at $50 \mathrm{~V}$ for $60 \mathrm{~min}$, denatured in $0.4 \mathrm{M} \mathrm{NaOH} / 1 \mathrm{M}$ Tris/HCl, $\mathrm{pH}, 7.0$, neutralized in $1 \mathrm{M} \mathrm{NaCl} / 1 \mathrm{M}$ Tris/HCl, pH, 7.4, blotted, UV-cross-linked, probed with the cognate ${ }^{32} \mathrm{P}$-radiolabeled full length cDNAs, radiographed at $-80^{\circ} \mathrm{C}$, and quantified densitometrically.

Dynamics of expression of HNF $1 \beta$ gene was studied at the selected time points (i.e., at $0,2 \mathrm{nd}$, 4th, 6th, 8th, 16th, 24th, 32nd, 48th, 72nd, and 96th h) by Relative RT-PCR. The experimental curve was plotted of (Fig. 3) on the basis of the relative absorbance obtained for each time point. Then, temporal fractal dimension was calculated for the experimental curve of the gene expression at three time intervals $0-8 \mathrm{~h}, 8-32 \mathrm{~h}$, and $48-96$ 
h. Since the curve is decreasing in the latter two time intervals, fractal dimension was also calculated for the "mirror" image of the curve.

All genes studied are known to be retinoid-responsive genes. Those genes contain retinoic acid responsive elements within their promoter regions, and are expected to be upregulated rather than downregulated in response to treatment with all-trans retinoic acid, either in in vitro or in vivo systems. The following primers and number of cycles were used:

RAR $\alpha$, Sense (S): GTA CCC AGT ACC CCC CTA CG, Antisense (A): GAC TCC TTG GAC ATG CCC AC, 20 cycles; RAR $\beta$, S: CAT CGA GAC ACA GAG TAC CA, A: GCT CTC TGT GCA TTC CTG CT, 20 cycles; RAR $\gamma$, S: ACC TCC TCG GGT CTA TAA GC, A: CGC AAA CTC CAC AAT CTT GA, 20 cycles; CRABP II, S: ATG AAT TCG GAG ACA GCA AAG TAT CTT TA, A: ATA AGC TTA AAT CAC ACA GAC TAC AAG G, 20 cycles; GATA4, S: CTG TAT GTA ATG CCT GCG G, A: AAG GTG GGC TTC TCT GTA A, 20 cycles; HNF 1 $\beta$, S: CTT CGA CAA TCA GTC ACC AT, A: AGC CAC ACT GTT AAT GAC CG, 30 cycles; HOX A-1, S: CTA CTT ACC AGA CTT CTG GA, A: CAA AGG TCT GCG CTG GAG AA, 18 cycles; HOX B-1 S: TAC CTG AGC CGT GCC CGG AG, A: GCC TTC CTT CGA CCT CTG AT, 18 cycles; MASH 1, S: AAG ACG ATC ACC CAG GCA, A: TGC CTG GGT GAT CGT CTT, 31 cycles; STRA 1, S: CGT GTC CTG GAG CTC TCT TA, A: CTT GAC AGT GTT GTC TGA CT, 18 cycles; STRA 6, S: CTT GTG CAG AGT CTC CGT CA, A: GGA CTA GAC CAG ACG TGA GA, 25 cycles; STRA 13, S: GCA CTT AGT AGA GTC TGA GAA AGG GCT G, A: ATG GAA CGG ATC CCC AGC GC, 20 cycles; 36B4, S: ATG TGA AGT CAC TGT GCC AG, A: GTG TAA TCC GTC TCC ACA GA, 18 cycles.

Morphometric analysis. Cells were recorded in a visual field of the microscope by the semiautomatic computerized image acquisition and analysis system consisting of the light microscope (Nikon, U.S.A.) conjugated with CCD camera (Hitachi, Japan), and image software package (Adobe Systems, U.S.A.) with frame graber of 512 $\times 512$ pixels installed upon PC computer. The radius of the most inner circle of the family of the concentric circles applied as a grid was $20 \mu \mathrm{m}$. It was chosen in such a way that the circle covered at least one cell located close to the center of the visual field. If a cell was cut by a circle into two parts, the cell was ascribed to the smallest circle covering its entire area, and counted only once.

Statistical analysis. Fractal dimension was calculated for each sample of cells from the radial distribution of the cells in the sample. We fitted numerical experimental data to the equation of nonlinear regression $\mathrm{y}=\mathrm{ax}^{\mathrm{b}}$, in which $\mathrm{y}$ is a number of cells (or, in the case of HNF $1 \beta$ expression, relative absorbance) within a circle of the grid with a given radius $\mathrm{x}$ (or time), $\mathrm{a}$ is a scaling coefficient, and $\mathrm{b}$ is fractal dimension (Sigma Plot ver. 4.0). We evaluated the $\mathrm{b}$ parameter together with its $\sigma$ standard error.

The significance of difference between the statistical groups was compared according to unbiased test of $\mathrm{F}$ statistics and ANOVA algorithm based upon Tukey $\mathrm{B}$ test at the confidence value $\alpha=$ 0.05 , and the critical value of $\mathrm{F}_{\alpha}=4.492$ (Microsoft Excel ver. 5.0).

\section{RESULTS}

Retinoid signal evokes expression of retinoid-responsive genes in P19 ${ }^{\text {mono }}$ or RAC65 ${ }^{\text {mono }}$ cells

Figure 1 shows the results of expression of retinoid-responsive genes, $\operatorname{RAR} \alpha, \beta, \gamma$, CRABP II, GATA4, HNF1 $\beta$, Hox A-1, Hox B-1, Mash I, Stra 1 , Stra 6, and Stra 13 in P19 mono and RAC65 ${ }^{\text {mono }}$ cells studied by Relative RT-PCR. The constitutive expression of all those genes in control, retinoid-sensitive P19 ${ }^{\text {mono }}$ cells was consistently lower than the constitutive expression of the same genes in control, retinoid-resistant RAC65 ${ }^{\text {mono }}$ cells with the exception of CRABP II, Hox A-1, and Stra 6 gene. The latter genes were expressed in RAC65 ${ }^{\text {mono }}$ cells below the level of detection of the method. The same cells treated with $100 \mathrm{nM}$ atRA responded with increased expression of all genes. That expression achieved the maximum of intensity in both cell populations usually about the 48 th $\mathrm{h}$ of treatment. 
RAR $\alpha$
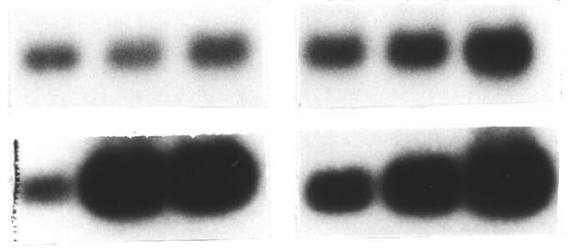

$\operatorname{RAR} \beta$
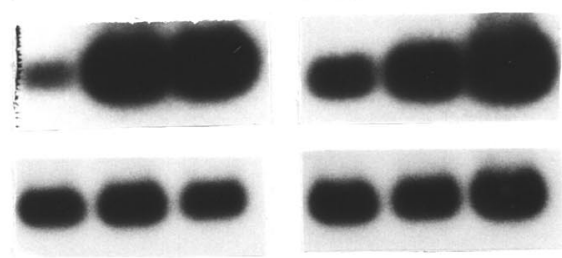

$\operatorname{RAR} \gamma$

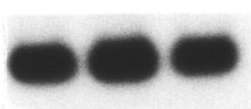

CRABP II

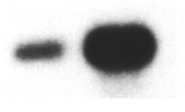

GATA4
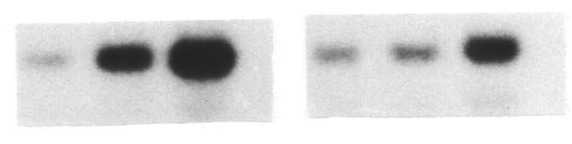

HNF $1 \beta$

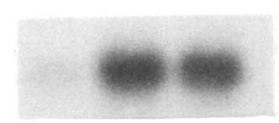

HOX A-1

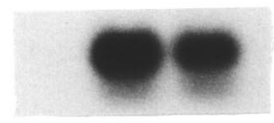

HOX B-1
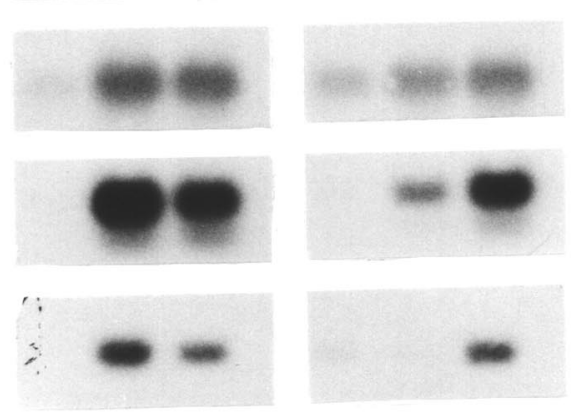

MASH I

STRA 1

STRA 6

STRA 13

36B4
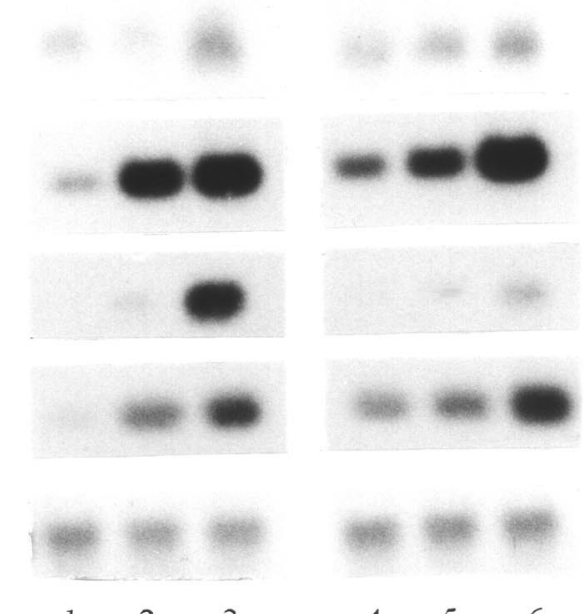

123

456

Figure 1. Results of Relative RT-PCR for expression of a series of retinoid-responsive genes, normalized against phosphoprotein $36 \mathrm{~B} 4$ in $\mathrm{P19}^{\text {mono }}$ cells (lanes 1-3) and RAC65 ${ }^{\text {mono }}$ cells (lanes 4-6).

Lane 1, control P19 ${ }^{\text {mono }}, \mathrm{P} 19^{\text {mono }}$ cells treated with 100 nM atRA for, (2) $24 \mathrm{~h}$, (3), $48 \mathrm{~h}$, (4), control RAC65 ${ }^{\text {mono }}$ cells, RAC65 ${ }^{\text {mono }}$ cells treated with $100 \mathrm{nM}$ atRA for (5) $24 \mathrm{~h}$, (6) $48 \mathrm{~h}$. The specific radioactivity of the appropriate probes was about 10 c.p.m./ $\mu \mathrm{g}$ DNA. Time of exposure of blots to X-ray films was approximately the same and not longer than $20 \mathrm{~min}$.
Dynamics of expression of retinoid-responsive genes is slower in RAC65 ${ }^{\text {mono }}$ cells than in P19 $^{\text {mono cells }}$

The retinoid-induced cellular expression of the mRNAs in the monolayer culture was increased from a few-fold to several hundred-fold in comparison with the constitutive expression. In the case of the following mRNAs: RAR $\beta$, RAR $\gamma$, CRABP II, GATA4, Stra 1, Stra 6, and Stra 13, the increased expression could be observed after the 24th $\mathrm{h}$ of retinoid treatment in $\mathrm{P} 19^{\text {mono }}$ cells. Simultaneously, expression of those mRNAs in RAC65 ${ }^{\text {mono }}$ cells, although usually greater than the constitutive one, was a few-fold lower than in $\mathrm{P} 19^{\text {mono }}$ cells in the 24 th $\mathrm{h}$ and reached comparable amounts later (i.e., in the 48th $\mathrm{h}$ of retinoid treatment) (Fig. 1). Only expression of RAR $\alpha$, Hox B-1, and Stra 13 mRNAs increased faster in RAC65 ${ }^{\text {mono }}$ cells than in $\mathrm{P} 19^{\text {mono }}$ cells.

\section{Dynamics of gene expression becomes diversi- \\ fied in cells undergoing aggregation and retinoid-induced differentiation}

Figure 2 illustrates expression of the same retinoid-responsive genes in $\mathrm{P} 19^{\text {agg }}$ cells and RAC65 $5^{\text {agg }}$ cells (i.e., cells treated with $1 \mathrm{nM}, 10$ $\mathrm{nM}$ or $100 \mathrm{nM}$ atRA during a $96 \mathrm{~h}$ period of aggregation and intercellular co-operation in colonies preceding initiation of neuronal or mesenchymal differentiation) as a function of time (i.e., in the 48 th $\mathrm{h}$ or in the 72th $\mathrm{h}$ of differentiation counted since the aggregated cells had been plated out onto wells).

The aggregation and intercellular co-operation changed the intensity of gene expression. The constitutive expression of the retinoid-responsive mRNAs in the P19 ${ }^{\text {agg }}$ or RAC65 $5^{\text {agg }}$ system was significantly different in comparison with expression of the genes in the $\mathrm{P} 19^{\text {mono }}$ or RAC65 ${ }^{\text {mono }}$ system. While constitutive expression of the genes in $\mathrm{P} 19^{\text {agg }}$ cells was at the approximately the same or greater level than in $\mathrm{P} 19^{\text {mono }}$ cells except $\operatorname{RAR} \beta$, the constitutive amounts of the same mRNAs were reduced in RAC65 $5^{\text {agg }}$ cells in comparison with RAC65 ${ }^{\text {mono }}$ cells with the exception CRABP II, Hox B-1, and Mash I genes, and in comparison with $\mathrm{P} 19^{\text {agg }}$ cells except Hox B-1 


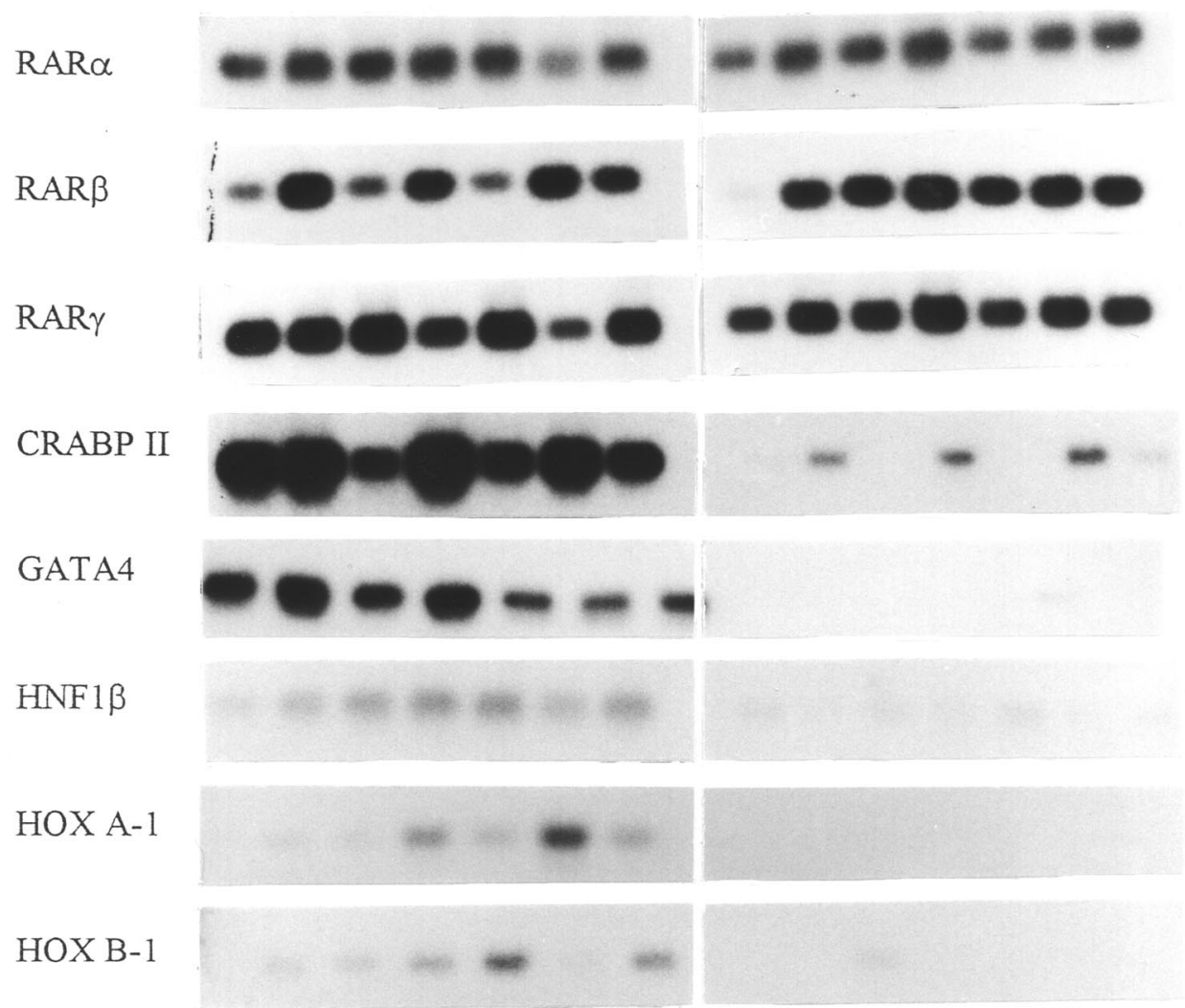

MASH I

STRA 1

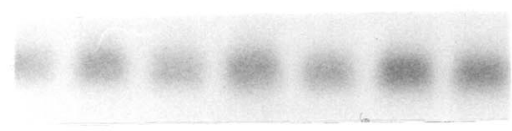

STRA 6

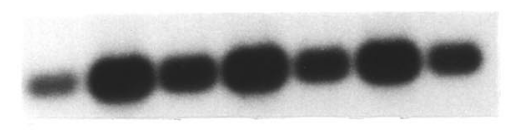

STRA 6

STRA 13

36B4
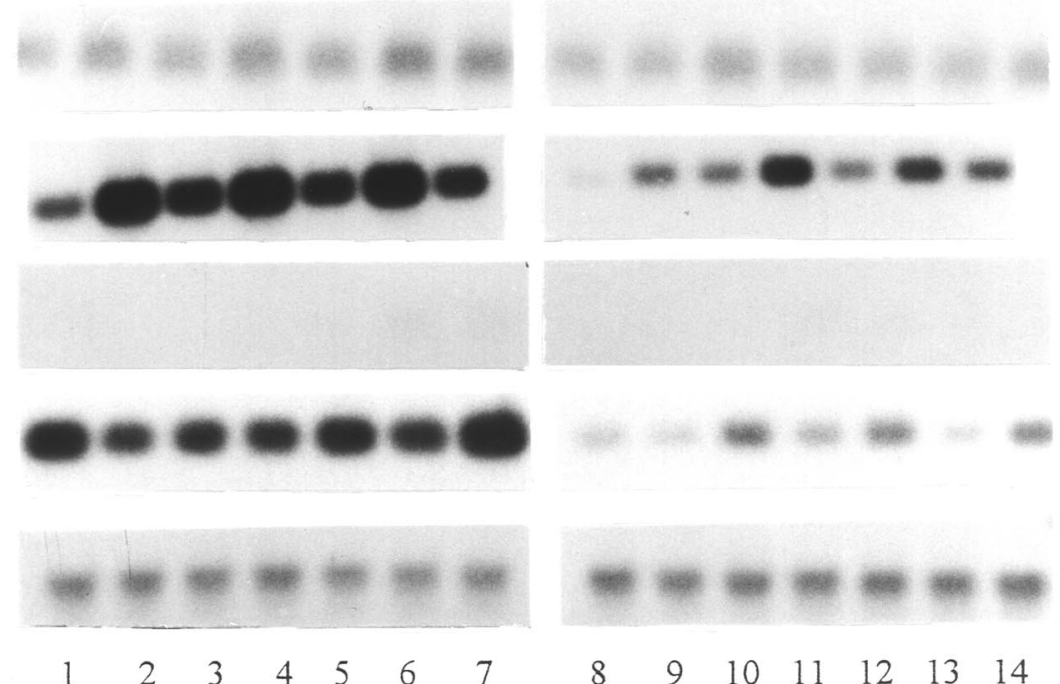

Figure 2. Results of Relative RT-PCR for expression of a series of retinoid-responsive genes, normalized against phosphoprotein mRNA 36B4 in P19 ${ }^{\text {agg }}$ cells (lanes 1-7) and RAC65 ${ }^{\text {agg }}$ cells (lanes 8-14).

Lane 1, control P19 ${ }^{\text {agg }}$ cells, P19 ${ }^{\text {agg }}$ cells treated with $1 \mathrm{nM}$ atRA, (2) $48 \mathrm{~h}$ after aggregation and retinoid treatment (AGRT), (3) $72 \mathrm{~h}$ after AGRT, P19 ${ }^{\text {agg }}$ cells treated with $10 \mathrm{nM}$ atRA, (4) $48 \mathrm{~h}$ after AGRT, (5) $72 \mathrm{~h}$ after AGRT, P19 cells treated with $100 \mathrm{nM}$ atRA, (6) $48 \mathrm{~h}$ after AGRT, (7) $72 \mathrm{~h}$ after AGRT. Lane 8, control RAC65 ${ }^{\text {agg }}$ cells, RAC65 ${ }^{\text {agg }}$ cells treated with $1 \mathrm{nM}$ atRA, (9) $48 \mathrm{~h}$ after AGRT, (10) $72 \mathrm{~h}$ after AGRT, RAC65 ${ }^{\text {agg }}$ cells treated with $10 \mathrm{nM}$ atRA, (11) $48 \mathrm{~h}$ after AGRT, (12) $72 \mathrm{~h}$ after AGRT, RAC65 ${ }^{\text {agg }}$ cells treated with $100 \mathrm{nM}$ atRA, (13) $48 \mathrm{~h}$ after AGRT, (14) $72 \mathrm{~h}$ after AGRT. The specific radioactivity of the appropriate probes was about $10^{9}$ c.p.m./ $\mu \mathrm{g}$ DNA. Time of exposure of blots to X-ray films was approximately the same and not longer than $20 \mathrm{~min}$. 
gene. Simultaneous reduction of gene expression in both $\mathrm{P} 19^{\text {agg }}$ and RAC65 ${ }^{\text {agg }}$ cells in comparison with the expression of those genes in $\mathrm{P} 19^{\text {mono }}$ or RAC65 $5^{\text {mono }}$ cells occurred in the case of $\operatorname{RAR} \beta$ and HNF $1 \beta$ mRNAs.

Aggregation and treatment of P19 cells with all-trans retinoic acid modified the dynamics of gene expression. While in P19 ${ }^{\text {mono }}$ cells the patterns of expression reflect simple up-regulation of retinoid-responsive genes, the patterns of expression in the same cells undergoing both aggregation and retinoid treatment are more complex. Consistently, expression of all retinoid-responsive genes was much lower or utterly shutt off in atRA-treated RAC65 ${ }^{\text {agg }}$ cells in comparison with the same cells cultured in the monolayer system. Dynamics of gene expression was damped in the aggregated RAC65 cells.

\section{Gene expression in time is a non-linear process}

Dynamics of this process differs from one time point to another, and there is no proportional relationship between input and the outcome (Fig. 3). It also is sensitive to the initial conditions (compare Figs. 1 and 2). Fitting of the experimental curve (Fig. 3 ) by equation $y(t)=a t^{b}$, in which $t$ denotes time, the exponent $b$ is fractal dimension in all three time intervals studied, shows that each out of the three time intervals possesses a

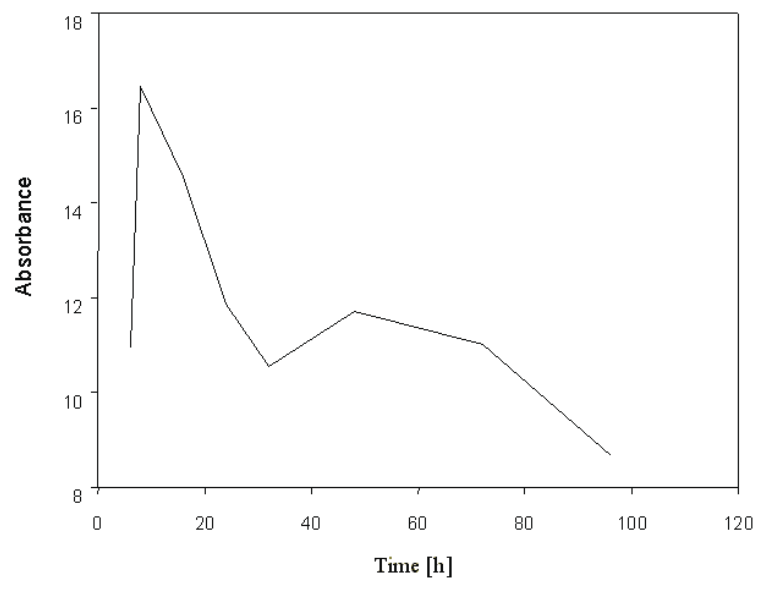

Figure 3. Dynamics of expression of the retinoid-responsive gene, HFN1 $\beta$ in $\mathrm{P}^{\text {mono }}$ cells treated with $100 \mathrm{nM}$ atRA.

Expression of the gene was measured by Relative RT-PCR, a semiquantitative method, in the 0 , 2nd, 4th, 6 th, 8 th, 16th, 24th, 32nd, 48th, 72nd, and 96th h. The experimental curve can be divided into three intervals, $0-8$ $\mathrm{h}, 8-32 \mathrm{~h}$, and $48-96 \mathrm{~h}$. The curve can be approximated in the first two intervals with equation $y(t)=a t^{b}$, in which $t$ denotes time, $b$ is fractal dimension at the value of coefficient of nonlinear regression $\mathrm{R}>0.95$ (cf. Table 2).

RAC65 $5^{\text {mono }}$ cells is slowed down in comparison with $\mathrm{P} 19^{\text {mono }}$ cells. This results in much worse fitting of the corresponding curve with $\mathrm{R} \ll 0.95$ and no fractal dimensions can be calculated (not shown). Since expression of the gene is shutt off in RAC65 ${ }^{\text {agg }}$ cells (Fig. 2), no curve can be plot-

Table 1. Fitting of the experimental curve for $P 19^{\text {mono }}$ cells (Fig. 3) by the equation $y(t)=a t$, in which $t$ denotes time, the exponent $b$ is fractal dimension in all three time intervals. $R$ is a coefficient of nonlinear regression, s denotes standard deviation.

The negative value of fractal dimension in the second and the third interval results from the fact that the experimental curve in those intervals decreases with time. Therefore, calculations "M" were also performed for the "mirror" image of the curve in those intervals.

\begin{tabular}{lccccc}
\hline Time interval $[\mathrm{h}]$ & \multicolumn{1}{c}{$\mathrm{a}$} & $\sigma$ & $\mathrm{b}$ & $\sigma$ & Coefficient of nonlinear regression R \\
\hline $0-8$ & 0.411 & 0.176 & 1.784 & 0.216 & 0.993 \\
$8-32$ & 31.820 & 4.720 & -0.306 & 0.054 & 0.969 \\
$48-96$ & 52.720 & 40.50 & -0.382 & 0.184 & 0.901 \\
$8-32 \mathrm{M}$ & 4.895 & 0.901 & 0.343 & 0.060 & 0.974 \\
$48-96 \mathrm{M}$ & 1.733 & 0.837 & 0.423 & 0.112 & 0.968 \\
\hline
\end{tabular}

fractal dimension. As can be seen from Fig. 1, dynamics of expression of HNF1 $\beta$ gene in ted, and, obviously, there is no temporal fractal dimension for that particular molecular process. 
Only differentiating $P 19^{\text {agg }}$ cells, but not P19 ${ }^{\text {mono }}$ cells, change their fractal dimension

Table 1 summarizes mean values of the fractal dimension obtained for the four cellular systems studied (i.e., P19 ${ }^{\text {mono }}, \mathrm{P} 19^{\mathrm{agg}}$, RAC65 ${ }^{\mathrm{mono}}$, and RAC65 ${ }^{\text {agg }}$ ). The coefficient of nonlinear regression $R>0.99$ for all $\mathrm{P} 19$ populations, $R<<0.95$ for all RAC65 populations.

The only statistically significant difference of fractal dimension was observed between control $\mathrm{P} 19^{\text {agg }}$ cells and $\mathrm{P} 19^{\text {agg }}$ cells differentiating under treatment with $100 \mathrm{nM}$ atRA during the $96 \mathrm{~h}$ period of aggregation (ANOVA; $p=2.4 \times 10^{-9}$ ). No difference was found between the following pairs: control P19 mono cells/atRA-treated P19 ${ }^{\text {mono }}$ cells, control RAC65 ${ }^{\text {mono }} /$ atRA-treated RAC65 $5^{\text {mono }}$, and control RAC65 $5^{\text {agg }} /$ atRAtreated RAC65 ${ }^{\text {agg }}$. Initially, control P19 ${ }^{\text {mono }}$ cells possessed a lower fractal dimension than control chical, self-similar structure of the pine tree type can be seen under the microscope [24]. However, the fractal structure of proliferating cells could be identified easily if both the cellular compartment and cell-free surface were analyzed by the box counting algorithm. Yet, the existence of nonlinear, exponential relationship between a number of P19 cells in the series of concentric circles and their radii, which increases in a linear manner, indicates that experimental data fit well to the power law scaling model of fractal $(\mathrm{R}>0.99, \mathrm{n}=$ 100 samples). All populations of $\mathrm{P} 19$ cells possess both a fractal dimension and self-similarity. This indicates that P19 cells form an interactive dynamic system.

A power-law property holds only for a limited range of scales. Therefore, efforts must be made to ensure that the experiment is performed within the proper interval of the independent variable. In

Table 2. The mean values of fractal dimension with (standard deviation), and range obtained for the P19/RAC65 cellular model

\begin{tabular}{lcc}
\hline Experimental system & Fractal dimension b & Range \\
\hline $\mathrm{P} 19^{\text {mono }}$ control & $1.856(92)$ & $1.780(90)-1.953(72)$ \\
$\mathrm{P} 19^{\text {mono }}$ atRA & $1.820(11)$ & $1.622(10)-1.953(66)$ \\
$\mathrm{P} 19^{\text {agg }}$ control & $2.144(10)$ & $1.923(10)-2.327(13)$ \\
$\mathrm{P} 19^{\text {agg }}$ atRA & $1.477(98)$ & $1.316(91)-1.635(89)$ \\
$\mathrm{RAC65}^{\text {mono }}$ control & $2.000(11)$ & $1.854(10)-2.211(15)$ \\
$\mathrm{RAC65}^{\mathrm{mono}}$ atRA & $1.905(11)$ & $1.757(11)-2.086(76)$ \\
$\mathrm{RAC65}^{\text {agg }}$ control & $1.994(12)$ & $1.846(14)-2.183(11)$ \\
$\mathrm{RAC65}^{\text {agg }}$ atRA & $2.057(11)$ & $1.833(70)-2.329(14)$ \\
\hline
\end{tabular}

RAC65 ${ }^{\text {mono }}$ cells (ANOVA; $p=0.0005$ ) which corresponds to lower growth rate of $\mathrm{P} 19$ cells. No difference was found between atRA-treated counterparts. The fractal dimension of $\mathrm{P} 19^{\text {agg }}$ cells was increased significantly, much above the values obtained either for P19 ${ }^{\text {mono }}$ cells or RAC65 ${ }^{\text {agg }}$ cells.

\section{DISCUSSION}

According to the definition given by Benoit Mandelbrot, the real image of a cellular population does not seem to be fractal because no hierar- the case of this work, the number of cells was measured in the family of concentric circles covering most of the visual field of the microscope. In each case out of 100 independent measurements, a non-integer value of the coefficient $b$ was found. This number of independent measurements creates a sufficiently large statistical group to obtain reliable statistics with high values of regression coefficient close to 1 . The mean value of the fractal dimension changed as cells underwent spatial transformations or transduced the retinoid signal; yet, differences between statistical groups or variability within those groups remained 
within a certain range of values, suggesting that the choice of the scale was correct (i.e., within the range of values at which the power-law does hold). The statistical data support the conclusion that the population of P19 cells reveals self-similarity; a prerequisite condition for restoration of dynamics of the entire system starting from a single P19 cell. The dimension of all populations of RAC65 cells equals the integer value of 2 within the limits of statistical error, and is independent of experimental conditions ( $\mathrm{R} \ll 0.95, \mathrm{n}=100$ samples). Therefore, the populations of RAC65 cells cannot be considered as fractals with an integer dimension. No self-similarity exists in the populations of RAC65 cells, either.

Dynamic processes (i.e., processes which change in the course of time), with a fractal dimension and self-similarity form an important class of physical phenomena. Their features are universal enough to hold for biological systems, such as cells or tissues. In the context of this study, if fractal space, in which a dynamic process takes place, becomes a classic, (i.e., Euclidean) space with integer dimension, this means that the process has lost stability, left its strange attractor (i.e., a fragment of fractal space in which the process occurs, and no further evolution of the system in time is possible unless extrasystemic forces act upon it), and tends towards, or already is in, a novel, local, and degenerated stationary state (i.e., the state with a lower number of possible directions of further evolution) [4].

In particular, loss of fractal dimension by a cellular system implies loss of intercellular connectivity (i.e., interconnectedness which denotes the existence of complex, dynamic relationships, not just structural, static ones, in a population of cells leading to the emergence of global features in the system that would have never appeared in a single cell existing out of the system), and loss of collectivity (i.e., capability of the interconnected cells to interact in a common mode) [10]. Such cells attain a number of unstable stationary states (heterogeneity) until they find novel attractors or degenerated stationary states associated with the emergence of unique phenotypes.

From the perspective of molecular reductionism, events associated with cellular prolifera- tion, such as gene expression [25, 26], passing of the restriction point $R$ in the cell cycle [27], or differentiation during migration along the intestinal crypt axis [28] are of stochastic nature (i.e., the phenomenon is determined by random action of extrasystemic forces). However, our analysis indicates that proliferation or differentiation occurs in the fractal subspace of Euclidean space-time. This fractal subspace is actively conquered, and, then, self-organized by the population of interacting cells $[8,9]$. Cellular phenomena are not limited to a series of stochastic events exclusive for each single cell. Rather, they are collective phenomena comprising the entire population as long as the cells are interconnected functionally. Connectivity enables cells to self-organize into hierarchical structures of the higher order, either geometric or energetic (e.g., compartmentalization) $[29,30]$. It is worth to notice that the discrepancy between the stochastic and the chaotic deterministic model exemplifies the relativity of findings resulting from the application of two different research approaches, reductionist and holistic.

Both $\mathrm{P} 19^{\text {mono }}$ and RAC65 ${ }^{\text {mono }}$ cells showed increased expression of retinoid-responsive genes (Fig. 1). The appropriate molecular systems transducing the retinoid signal were active in both cell types. Yet, aggregation initiated differentiation of P19 cells only. During differentiation, constitutive gene expression was decreased, and dynamics of gene expression was altered in comparison with $\mathrm{P} 19^{\mathrm{mono}}$ cells. Also, the fractal dimension $\mathrm{P} 19^{\text {agg }}$ cells treated with atRA (i.e., during transition from the phase of proliferation to differentiation) was decreased. Most likely, differentiation of $\mathrm{P} 19^{\text {agg }}$ cancer cells requires more than just simple expression of a number of phenotype-specific genes [14, 16, 17]. It also requires temporal and spatial organization of those molecular events (i.e., the appropriate dynamics). Indeed, a population of P19 cells "remembers" aggregation in suspension as an impulse altering the dynamics of inter- and intracellular events (compare Figs. 1 and 2). The changes of intracellular dynamics appear to be related to the changes of both spatial and temporal fractal dimension. Indeed, we demonstrated that a relationship between expression of at least some genes and time can be non-linear 
(see Fig. 3). Such a non-linear curve can be approximated in appropriate time intervals by exponential function with $R>0.95$ (Table 2), in which the exponent is fractal dimension. In general, dynamics of gene expression could be very complex, and such approximation might require more sophisticated fitting. It appears, however, that not only the space occupied by the system of interacting cells has a fractal dimension, but also has the time in which cellular events do occur. It is, therefore, necessary to consider the influence of geometry of space-time upon molecular cellular phenomena, such as gene expression or phenotype formation. In particular, patterns of gene expression obtained from the experiments in vitro performed in the monolayer cell cultures cannot be interpolated anymore to quasi-threedimensional normal tissues or tumors in vivo. For the same reason, one must be very careful while interpreting results of genetic experiments in which techniques such as gene knockout or gene transfection were applied to achieve any phenotypic effect [1].

Dimensions of all RAC65 populations equal 2. Despite aggregation, RAC65 ${ }^{\text {agg }}$ cells do not differentiate. Dynamics of gene expression in RAC65 ${ }^{\text {agg }}$ cells was damped in comparison with RAC65 ${ }^{\text {mono }}$ cells or with $\mathrm{P} 19^{\text {agg }}$ cells (e.g., CRABP II, GATA4, Hes I (not shown), Hox A-1, Hox B-1, HNF1及, Stra 1, Stra 6, and Stra 13 mRNA). In addition, there is no temporal fractal dimension in the case of expression of HNF $1 \beta$ gene in RAC65 cells. That fact supports the view that RAC 65 cells have lost the space-time attractor typical of P19 cells. It is noteworthy that the difference between RAC65 ${ }^{\text {agg }}$ and RAC65 $5^{\text {mono }}$ cells or between RAC65 agg and P19 ${ }^{\text {agg }}$ cells relies upon the degree of complexity of the system. P19 cells underwent irreversible decomplexification during tumor progression. A novel dynamic network of feedbacks between genes and their regulatory elements emerged in RAC65 cells. RAC65 ${ }^{\text {mono }}$ cells respond to retinoid signal with simple up-regulation as $\mathrm{P} 19^{\text {mono }}$ cells do. However, the same RAC65 cells forced to interact in a more complex system, such as an aggregate, cannot restore the dynamics of gene expression which P19 cells can develop. Apparently, RAC65 cells attained during tumor progression a degenerated stationary state typical of non-interactive, deterministic systems. That state is characterized by the loss of both spatial and temporal fractal dimension, of self-similarity, connectivity, and collectivity. Those results suggest that tumor progression can eventually lead every population of primary cancer cells to such a degenerated stationary state which is associated with the metastatic phenotype.

To summarize, the following findings should be emphasized. First, a population of P19 cells possesses both fractal dimension and self-similarity. The co-existence of those traits denotes that each single cancer cell can restore the organization and identical dynamic features of the tumor. In particular, the dynamics of growth of malignant tumor at the organism level must be identical with the dynamics at the level of a single cell, and vice versa. Second, cellular phenomena, such as proliferation or differentiation, which occur in the hierarchical tissue structure having a fractal dimension, are collective. Third, fractal dimension changes along with transition of cells from proliferation phase to differentiation phase. Both statistical features (i.e., fractal dimension and self-similarity) are lost in the course of tumor progression. Tumor progression leads the population of primary cancer cells to the degenerated stationary state characterized by damped dynamics of gene expression, non-interactivity, loss of connectivity and collectivity. Fractal structure of both time and space in which a cellular system exists and performs its functions is necessary for self-organization to emerge.

We acknowledge the kind interest, discussion, and excellent technical support of Dr. R. Taneja of Mount Sinai School of Medicine in New York.

\section{RE F E R E N C ES}

1. Waliszewski, P., Molski, M. \& Konarski, J. (1998) On the holistic approach in cellular and cancer biology, nonlinearity, complexity, and quasi-deter- 
minism of dynamic cellular network. J. Surg. Oncol. 68, 70-78.

2. Hastings, H.M. \& Sugihara, G. (1993) Fractals. A User's Guide for the Natural Sciences; pp. 15-35, Oxford University Press, Oxford.

3. Baker, G.L. \& Gollub, J.P. (1996) Chaotic Dynamics, an Introduction; pp. 171-196, Cambridge University Press, New York.

4. Devaney, R.L. (1986) Introduction to Chaotic Dynamical Systems; pp. 176-202, Benjamin Cummings, Reading.

5. Peitgen, H.O., Jurgens, H. \& Saupe, D. (1992) Chaos and Fractals; pp. 192-225, Springer Verlag, Heidelberg.

6. Bassingthwaighte, J.B., Liebovitch, L.S. \& West, B.J. (1994) Fractal Physiology. Oxford University Press, Oxford.

7. Cross, S.S. (1997) Fractals in pathology. J. Pathol. 182, 1-8.

8. Losa, G.A. \& Nonnenmacher, T.F. (1996) Self-similarity and fractal irregularity in pathologic tissues. Modern Pathol. 9, 174-182.

9. Smith, T.G., Jr., Lange, G.D. \& Marks, W.B. (1996) Fractal methods and results in cellular morphology - dimensions, lacunarity, and multifractals. J. Neurosci. Meth. 69, 123-136.

10. Waliszewski, P., Molski, M. \& Konarski, J. (1999) Self-similarity, collectivity, and evolution of fractal dynamics during retinoid-induced differentiation of cancer cell population. Fractals 7, 139-149.

11. Bain, G., Ray, W.J., Yao, M. \& Gottlieb, D.J. (1994) From embryonal carcinoma cells to neurons, the P19 pathway. Bioessays 16, 343-348.

12. Edwards, M.K. \& McBurney, M.W. (1983) The concentration of retinoic acid determines the differentiated cell types formed by a teratocarcinoma cell line. Dev. Biol. 98, 187-191.

13. Horn, V., Minucci, S., Ogryzko, V.Y., Adamson, E.D., Howard, B.H., Levin, A.A. \& Ozato, K. (1996) RAR and RXR selective ligands cooperatively induce apoptosis and neuronal differentiation in P19 embryonal carcinoma cells. FASEB J. 10, 1071-1077.

14. Jones-Villeneuve, E.M., McBurney, M.W., Rogers, K.A. \& Kalnins, V.I. (1982) Retinoic acid induces embryonal carcinoma cells to differentiate into neurons and glial cells. J. Cell Biol. 94, 253-262.

15. Skerjanc, I.S., Slack, R.S. \& McBurney, M.W. (1994) Cellular aggregation enhances MyoD-directed skeletal myogenesis in embryonal carcinoma cells. Mol. Cell. Biol. 14, 8451-8459.

16. Staines, W.A., Morassutti, D.J., Reuhl, K.R., Ally, A.I. \& McBurney, M.W. (1994) Neurons derived from P19 embryonal carcinoma cells have varied morphologies and neurotransmitters. Neuroscience 58, $735-751$.

17. Staines, W.A., Craig, J., Reuhl, K. \& McBurney, M.W. (1996) Retinoic acid treated P19 embryonal carcinoma cells differentiate into oligodendrocytes capable of myelination. Neuroscience 71, 845-853.

18. Pratt, M.A., Kralova, J. \& McBurney, M.W. (1990) A dominant negative mutation of the alpha retinoic acid receptor gene in retinoic acid-nonresponsive embryonal carcinoma cells. Mol. Cell. Biol. 10, 6445-6453.

19. Pratt, M.A., Langston, A.W., Gudas, L.J. \& McBurney, M.W. (1993) Retinoic acid fails to induce expression of Hox genes in differentiation-defective murine embryonal carcinoma cells carrying a mutant gene for alpha retinoic acid receptor. Differentiation 53, 105-113.

20. Berg, R.W. \& McBurney, M.W. (1990) Cell density and cell cycle effects on retinoic acid-induced embryonal carcinoma cell differentiation. Dev. Biol. 138, 123-135.

21. Chomczynski, P. \& Sacchi, N. (1987) Single-step method of RNA isolation by acid guanidinum thiocyanate-phenol-chloroform extraction. Anal. Biochem. 162, 156-159.

22. Bouillet, P., Oulad-Abdelghani, M., Vicaire, S., Garnier, J.M., Schuhbaur, B., Dolle, P. \& Chambon, P. (1995) Efficient cloning of cDNAs of retinoic acid-responsive genes in P19 embryonal carcinoma cells and characterization of a novel mouse gene Stra 1 (monouse Lerk-2). Dev. Biol. 170, 420-433.

23. Krowczynska, A.M., Coutts, M., Makrides, S. \& Brawerman, G. (1987) The mouse homologue of the human acidic ribosomal phosphoprotein PO, a highly conserved polypeptide that is under translational control. Nucleic Acids Res. 17, 6408-6412. 
24. Mandelbrot, B. (1983) The Fractal Geometry of Nature. Freeman, New York.

25. Ko, M.S.H., Nakauchi, H. \& Takahashi, N. (1990) The dose dependence of glucocorticoid-inducible gene expression results from changes in the number of transcriptionally active templates. EMBO J. 9, 2835-2842.

26. Ko, M.S.H. (1991) A stochastic model for gene induction. J. Theor. Biol. 153, 181-194.

27. Campisi, J., Medrano, E.E., Morreo, G. \& Pardee, A.B. (1982) Restriction point control of cell growth by a labile protein, evidence for increased stability in transformed cells. Proc. Natl. Acad. Sci. U.S.A. 79, 436-440.

28. Potten, C.S. \& Loeffler, M. (1990) Stem cells, attributes, cycles, spirals, pitfalls, and uncertainties. Lessons from the crypt. Development 110, 1001-1020.

29. Cummings, F.W. (1985) A pattern-surface interactive model of morphogenesis. J. Theor. Biol. 116, $243-273$.

30. Nicolis, J.S. (1986) The concept of complexity; in Dynamics of Hierarchical Systems. An Evolutionary Approach (Nicolis, J.S., eds.) pp. 72-73, Springer Verlag, Berlin. 